\title{
Kniest Dysplasia with Severe Tracheomalacia: Bone Dysplasia with Respiratory Complication
}

\author{
Tadashi Moriwake ${ }^{1}$, Masako Kawakami-Shinozuka ${ }^{1}$, Masaharu Yasumitsu ${ }^{2}$ and Tetsuya Nakamura ${ }^{3}$ \\ ${ }^{1}$ Departments of Pediatrics, ${ }^{2}$ Orthopedic Surgery, and ${ }^{3}$ Radiology, Iwakuni Clinical Center, Yamaguchi, Japan
}

\section{Introduction}

Respiratory distress is one of the most serious life-threatening complications for patients with systemic bone disease. Reduced thoracic volume due to hypoplastic or deformed thorax, fragility of thorax, and narrowing or obstruction of the air way may cause respiration problems.

In the present case, the patient had been treated for chronic respiratory problems since 1 yr old. Bone deformity and growth retardation became apparent by $3 \mathrm{yr}$ old. Bone symptoms were initially recognized by odd-looking gait and contracture of PIP joints, and a final diagnosis of Kniest dysplasia with severe laryngothoracheomalacia was made.

\section{Case Presentation}

A male newborn infant was transferred to our neonatal intensive care unit (Iwakuni Clinical Center: ICC) because of feeding difficulty and apnea associated with cleft palate.

The feeding problem and apnea were treated by nasogastric tube feeding and oxygen supplementation. Respiratory status was stable until 1 yr 1 mo old.

The patient was suffered from bronchopneumonia with severe inspiratory

Correspondence: Dr. Tadashi Moriwake, Department of Pediatrics, Iwakuni Clinical Center, 2-5-1 Kuroiso-cho, Iwakuni, Yamaguchi 740-8558, Japan

E-mail: moriwake@iwakuni-nh.go.jp dyspnea at the age of 1 yr 1 mo old, and required intermittent mandatory ventilation. Without respiratory support, retraction stayed prominent and chest deformity with funnel chest became obvious. The patient was transferred to ICC again because of a further worsening of inspiratory dyspnea after an operation for cleft palate at the age of 2 yr 11 mo old.

Inspiratory dyspnea was apparent with severe substernal and intercostal retractions. Oxygen saturation remained low ( $\mathrm{SpO}_{2}: 92 \%$ with room air) and narrowing of the bronchus was obvious. The patient required oxygen supplementation for one month. Endoscopic observation did not reveal organic narrowing or tumor of the bronchus. Blood analysis for the immune system did not show any chronic illness or immune deficiency. Keeping a prone position was dramatically effective for treating the respiratory problem.

We considered that chronic bronchopneumonia with laryngotracheomalacia caused the severe inspiratory dyspnea. The patient was treated with continuous administration of bronchodilator and long-term prophylactic antibiotics administration. The treatment was successful and he could terminate continuous medication at the age of $4 \mathrm{yr} 1 \mathrm{mo}$.

At the age 4 yr 3 mo, odd-looking gait and contracture of finger joints became apparent. Growth curve was linear and mild growth retardation was noticed (Fig. 1). His height and weight were $95.3 \mathrm{~cm}(-1.9 \mathrm{SD})$ and $15.9 \mathrm{~kg}$, respectively. His father's and mother's heights 


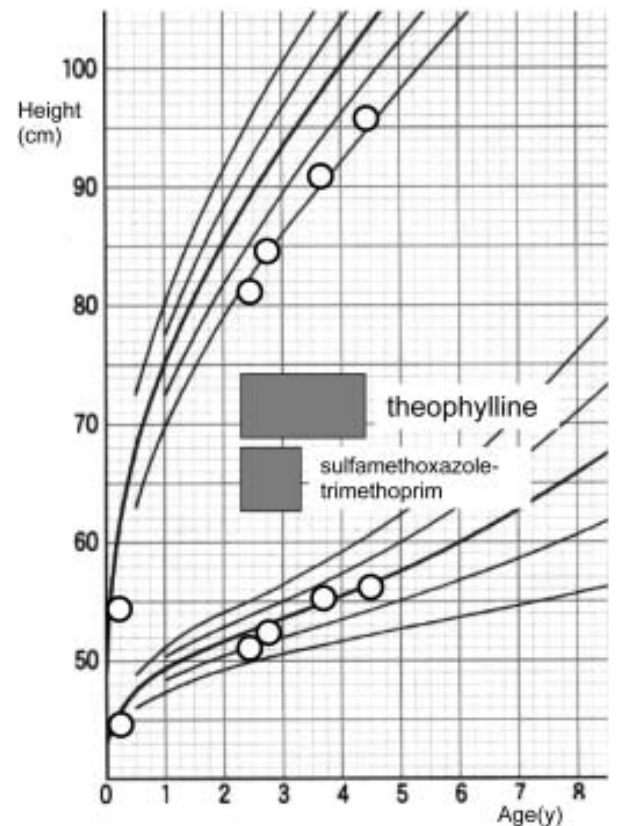

Fig. 1 Growth curve revealed mild growth retardation. were $163 \mathrm{~cm}$ and $155 \mathrm{~cm}$, respectively, and there was no family history of short stature or bone disease. The patient had a round flat face and mild chest deformity (funnel chest: Fig. 2). His knee and elbow joints showed prominent swelling. Flections of metacarpophalangeal joints of the hand were severely disturbed.

General psychomotor development was normal except for speech retardation.

Conventional blood biochemistry, blood cell counts, and urine analysis did not show any abnormalities.

Radiographic findings revealed widening and flaring of metaphyses and flat deformed epiphyses of the long bones, especially at the humerus, femur and tibia (Figs 3, 4, and 5). Odontoid process of axis was wide. Mild platyspondyly with deformed thoracic spine was noticed.

The ophthalmologic examination did not show any abnormalities, and hearing was not impaired.

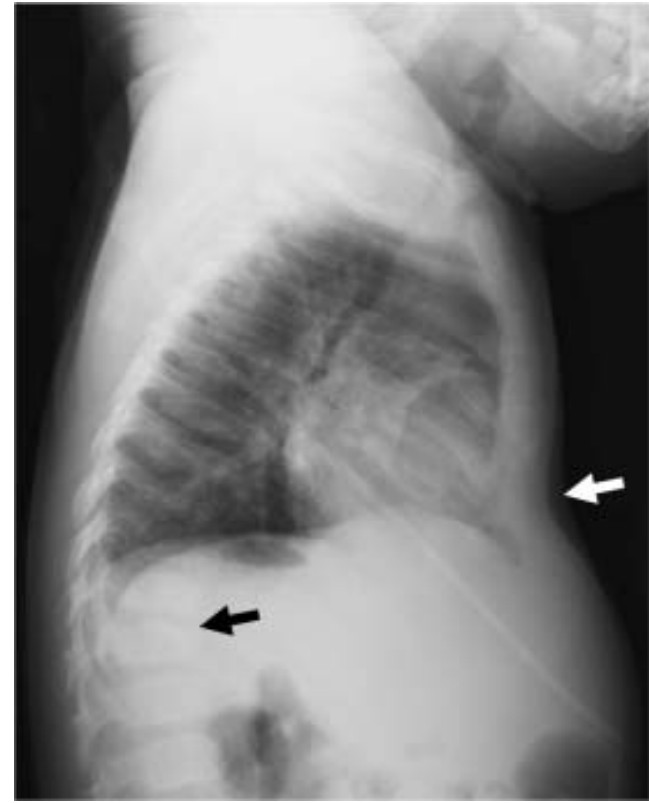

Fig. 2 Chest X-ray revealed funnel chest deformity (white arrow), flattened vertebral bones and deformity of thoracolumbar junction (black arrow).

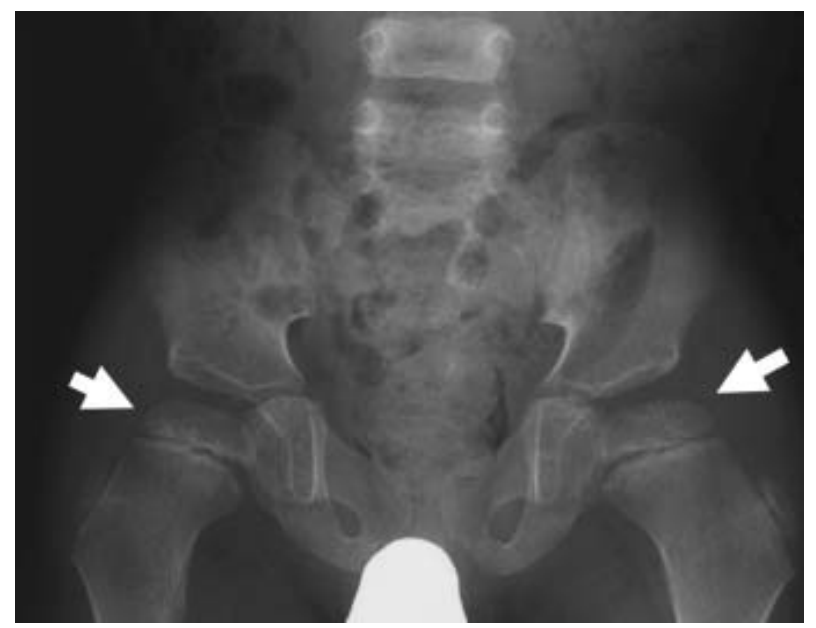

Fig. 3 Pelvic bone and proximal end of femur. Femoral epiphysis showed flattened deformity.

According to the summarized clinical feature with radiographic findings, we diagnosed the patient as suffering from Kniest dysplasia. 


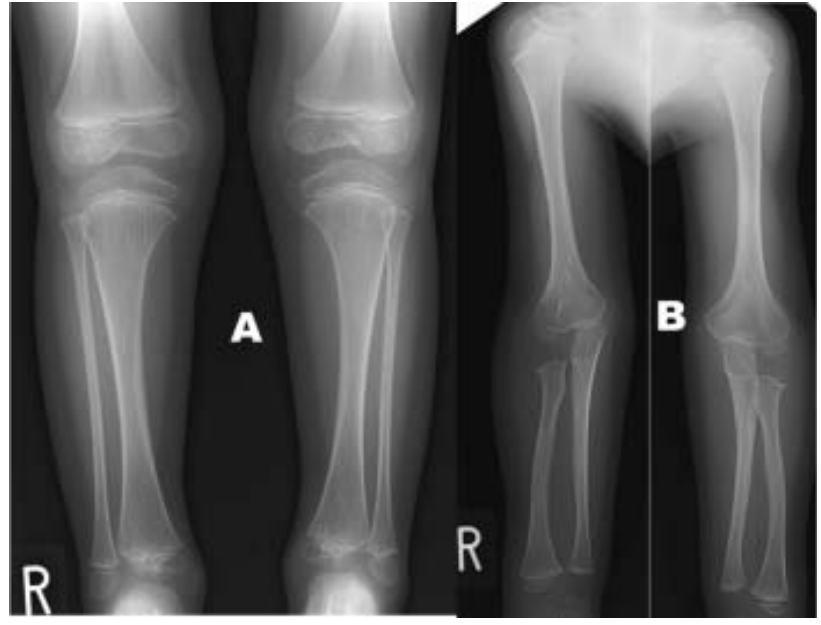

Fig. 4 A: lower legs, B: upper extremities. Knee and elbow joint were swollen with epiphyseal deformity.

\section{Discussion}

Kniest dysplasia (MIM \#156550) is a rare autosomal dominant inherited skeletal dysplasia characterized by metaphyseal flaring of long bones and progressive stiffness of joints. Mutations in the COL2A1 gene have been reported and pathological changes, mainly documented in cartilage, are reported as being Swiss cheese-like in appearance.

Skeletal changes demonstrate several characteristic features in shape such as large skull, round and flat face with depressed nasal bridge, cleft palate, short neck, lordosis and/or kyphosclerosis. Functionally, limitation of joint movement may be serious, such as incapability of making tight fist and altered gait because of deformity of the femoral head.

The extra-skeletal system shows several symptoms, especially observed in the eyes and auditory system. Myopia and chorioretinal thinning and sensorineural hearing impairment have been reported.

Respiratory distress is a common cause of death among patients with neonatal-lethal bone dysplasias. Respiratory problems are not

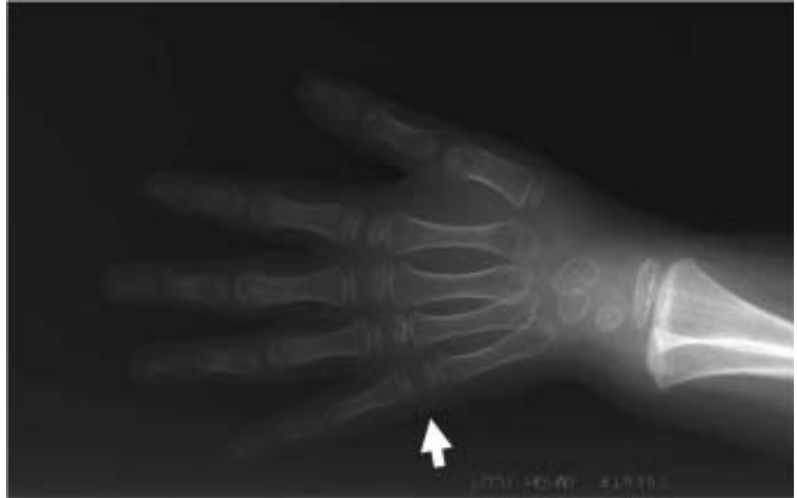

Fig. 5 Hand bones. Deformities of epiphyses are indicated (MP joints).

commonly reported for patients with Kniest dysplasia, except for a couple of reports of neonatal-lethal cases $(1,2)$. A decreased amount of type II collagen is not only observed in cartilage but also in the airway and may cause tracheomalacia.

Tracheomalacia or respiratory distress was reported in a neonatal lethal case with Kniest dysplasia $(1,2)$. Pathological changes were also observed in the tracheal cartilage as well as epiphyseal plate cartilage. The mutation in the type II collagen molecule may cause fragility of the tracheal structure. Reports of association of tracheomalacia with Kniest dysplasia are rare; however, the risk of association of dyspnea should be considered.

In the present case, the clinical symptom of dyspnea with retraction became obvious around 1 yr old. Bronchopneumonia was a trigger for a worsening respiration state. This was a late-onset but severe chronic dyspnea might have persisted because of association of fragility of the airway and narrowing of the airway caused by chronic inflammation. The combined treatment of theophylline, antibiotics, and keeping a prone position was successful. The effectiveness of the bronchodilator might suggest the involvement of bronchospasm in this patient's condition. Position change was dramatically effective for suppressing dyspnea; however, definite laryngomalacia was 
Table 1 Respiratory symptoms in skeletal dysplasias

\begin{tabular}{ll}
\hline $\begin{array}{l}\text { Narrowing of airway } \\
\text { Micrognathia }\end{array}$ & Pierre-Robin anomaly \\
$\begin{array}{l}\text { Tonsilar hypertrophy (relative) } \\
\text { Laryngothoracheomalacia }\end{array}$ & $\begin{array}{l}\text { achondroplasia } \\
\text { craniocarpotarsal dysplasia, } \\
\text { frontometaphyseal dysplasia }\end{array}$ \\
& Kniest dysplasia \\
Fragile thorax & osteogenesis imperfecta \\
Hypoplastic thoracic cavity (platyspondyly, scoliosis, thoracic deformity) & SED congenita, other spondylometaphyseal dysplasia \\
& Asphyxiating thoracic dysplasis \\
& Most of neonatal-lethal bone disease \\
Associated immune deficiency & McKusick metaphyseal chondrodysplasia \\
\hline
\end{tabular}

not identified by endoscopic examination. We considered the region responsible for retraction was tracheomalacia, which was resolved naturally by growth. Because of association of fragility of the thoracic cavity with inspiratory dyspnea, thoracic deformity became apparent as funnel chest.

The respiratory state has often been reported as a cause of death in neonatal life-threatening skeletal diseases. However, the severity of dyspnea varies between cases. Proposed causes of dyspnea and skeletal diseases are listed in Table 1. Airway obstruction/narrowing and small thorax are two major causes of dyspnea. Therapy for dyspnea may be required for severely affected cases.

\section{References}

1. Hicks J, De Jong A, Barrish J, Zhu SH, Popek E. Tracheomalacia in a neonate with Kniest dysplasia: histopathologic and ultrastructural features. Ultrastruct Pathol 2001;25:79-83.

2. Chen H, Yang SS, Gonzalez E. Kniest dysplasia: neonatal death with necropsy. Am J Med Genet 1980;2:171-8. 